\title{
Energy distribution among the reflected and refracted plane elastic waves at the boundary between transversely isotropic media
}

\author{
$2^{\text {nd }}$ Part
}

\section{Gulzar AHMAd}

\author{
Ricevuto il 15 Maggio 1967
}

RiAsSunto. - In questa seconda parte, vengono studiati i rapporti dell'energia fra onde riflesse e rifratte dalla superficie limitante due mezzi trasversalmente isotropi. L'equazione dell'energia è stata ricavata tenendo conto due volte dell'energia cinetica $(a)$, due volte dell'energia potenziale $(b)$.

I rapporti fra le onde originate e l'onda incidente quasilongitudinale, sono stati calcolati per il caso particolare in cui l'asse di simmetria coincide con la normale alla superficie limitante $i$ due mezzi in questione.

SUmmary. - The energy ratios of the reflected and refracted waves at the boundary between transversely isotropic media have been investig. ated. The energy equation has been derived on two bases, namely as (a) double of the kinetic energy, $(b)$ double of the potential energy. The ratios of the derived waves to that of the incident quasilongitudinal wave have been calculated for the particular case, where the symmetry axes of the media coincide with the normal to the boundary surface. The influence of varying the different elastic parameters is shown in a few diagrams.

Zusammenfassung. - Die Energieverhaeltnisse der an einer Grenzflaeche zwischen transversalisotropen Medien reflektierten und gebrochenen Wellen sind untersucht worden. Die Energiegleichung ist auf zweierlei Art aufgestellt worden, naemlich als (a) doppelte kinetische Energie, $(b)$ doppelte potentielle Energie. Die Energieverhaeltnisse der aufgespaltenen Wellen sind fuer die einfallende quasilongitudinale Welle fuer den Sonderfall berechnet worden, in dem die Symmetrieachsen beider Medien mit der Grenzflaechennormale zusammenfallen. In verschiedenen Diagrammen ist der Einfluss der Variation verschiedener elastischer-Parameter dargestellt. 
In this article the energy ratios of the derived waves would be investigated, when a plane elastic wave strikes a surface of discontinuity between two transversely anisotropic media.

As is generally known, in an elastic medium the total energy consists of (a) kinetic (b) potential energy. The latter is obtained by multiplying the stress tensor with the strain tensor, while the former is proportional to the square of the velocity of the particles in the medium. In elastic waves both of them averaged over a full period are equal. Accordingly there are two methods of calculating the total energy (or energy flux) in a wave, namely as twice the:
(a) kinetic energy,
(b) potential energy.

In the following the expressions for the ray velocity (the velocity of the energy propagation) and its direction as a function of the direction of the wave normals would be derived, since they would be needed for the calculation of the energy flux.

\section{Ray Direotion and Ray Velocity.}

It is a characteristic property of the wave propagation in the anisotropic media that the direction and the velocity of the propagation of the energy (ray energy or, with some authors, group velocity) deviate from the direction of the wave normals and the phase velocity respectively, see for example Lighthill $\left(^{1}\right)$. That the displacement vector also deviates from the ray or wave normal - direction can be seen from Eq. 9 of the last article $\left({ }^{2}\right)$.

Lighthill has extended an argument of Rayleigh $\left(^{3}\right)$ for calculating the group velocity to the three dimensional case as follows:

$$
\bar{U}=\operatorname{grad} G /(\partial G / \partial \omega)
$$

where:

$$
\begin{aligned}
& U=\text { the group or ray velocity, } \\
& \theta=\text { the slowness surface. }
\end{aligned}
$$

Rudzki (1911) has given a parameter expression for the calculation of the ray direction and velocity. Another method to calculate the ray direction as a function of the wave normals directly is to use the energy vector, that is, the vector which points in the direction of the 
energy propagation (ray direction); namely the following identity must hold for it:

$$
\operatorname{tg} \beta=\frac{\text { horizontal component of the flux }}{\text { vertical component of the flux }} \text {, }
$$

and the components of the energy flux can be obtained from Eq. [2] Love $\left({ }^{2}\right)$ :

$$
\begin{aligned}
& F_{x}=-\left(\dot{u} \cdot \tau_{x x}+\dot{v} \cdot \tau_{x y}+\dot{w} \cdot \tau_{x z}\right), \\
& F_{y}=-\left(\dot{u} \cdot \tau_{y x}+\dot{v} \cdot \tau_{y y}+\dot{w} \cdot \tau_{y z}\right), \\
& F_{z}=-\left(\dot{u} \cdot \tau_{x z}+\dot{v} \cdot \tau_{y z}+\dot{w} \cdot \tau_{z z}\right),
\end{aligned}
$$

where $u, v, w$ and $\tau_{i k}$ are the $x-, y-, z-$ components of the displacement vector and stress tensor, and the dots on them mean the differentiation with respect to time.

From [2] one gets:

$$
\operatorname{tg} \beta=\operatorname{tg} \varphi \frac{\alpha_{1} \sin ^{4} \varphi+\alpha_{2} \sin ^{2} \varphi+\alpha_{3}}{\alpha_{4} \sin ^{4} \varphi+\alpha_{5} \sin ^{2} \varphi+\alpha_{\theta}},
$$

where:

$$
\begin{aligned}
& a_{1}=L\left(\overline{A-L^{2}}-\overline{L+F^{2}}\right), \\
& \alpha_{2}=L\left(\overline{L+F)^{2}}+\left(L-\varrho V^{2}\right)\left[(L+F)^{2}+2 L(A-L)\right]\right. \text {, } \\
& \alpha_{3}=\left(L-\varrho V^{2}\right)\left[L\left(L-\varrho V^{2}\right)-\overline{L+F^{2}}\right] \text {, } \\
& a_{4}=C(\overline{A-L})^{2}-(\overline{L+F})^{2} A \text {, } \\
& \alpha_{5}=L(L+F)^{2}+\left(L-\varrho V^{2}\right)\left[2 C(A-L)-(L+F)^{2}\right] \text {, } \\
& a_{0}=C\left(L-\varrho V^{2}\right)^{2} \text {. }
\end{aligned}
$$

The Energy Flux: its calculation from the inetio energy.

In the following it would be assumed that the ray velocity and its direction are known, since they can be calculated quite easily from Eq. 3 and (6) (see below).

\section{Waves of $I$ or II type.}

As in the last article $\left({ }^{2}\right)$ the quasilongitudinal and quasitransversal waves in the anisotropic media are termed here the waves of the I and II type respectively. Owing to the very similar (almost identical) form of their representation through a plane wave, they can be considered here together. The energy flux of the individual waves may 
be calculated separately and then added together to give the total energy $\left({ }^{5}\right)$. A plane wave of both types can be written as follows:

$$
\begin{aligned}
& u=-G \frac{L+F}{N(\varphi)} \sin \varphi \cos \varphi \cos \omega\left(\frac{x \sin \varphi+z \cos \varphi}{V}-t\right) \\
& w=\frac{G}{N(\varphi)}\left(\overline{A-L} \sin ^{2} \varphi+L-\varrho V^{2}\right) \cos \omega\left(\frac{x \sin \varphi+z \cos \varphi}{V}-t\right),
\end{aligned}
$$

with

$$
\left.N^{2}(\varphi)=(L+F)^{2} \sin ^{2} \varphi \cos ^{2} \varphi+\overline{(A-L} \sin ^{2} \varphi+L-\varrho V^{2}(\varphi)\right)^{2} .
$$

The kinetic energy per unit volume is given by:

$$
F=E V, S
$$

where:

$$
\begin{aligned}
& F=\text { the energy flux, } \\
& E=\text { the total energy (= twice the kinetic), } \\
& V_{S}=\text { the ray velocity, } \\
& S=\text { the surface of the wave front, which takes part in the } \\
& \quad \text { reflexion and transmission process. }
\end{aligned}
$$

The energy flux per unit surface of the boundary surface would now be calculated. From Fig. 1 it is clear that the energy contained in the wave surface $\overline{A C}$ propagates along $\overline{A^{\prime \prime} A}-\overline{B^{\prime \prime} B}$. Imagine a bundle of rays and follow its path. It would distribute itself on $\overline{A B}$ of the boundary surface. One has:

where

$$
\begin{aligned}
A D & =A B \cos \beta, \\
& =A C \cos (\beta-\varphi),
\end{aligned}
$$

$$
\begin{aligned}
& \beta=\text { the ray direction }, \\
& \varphi=\text { the direction of the wave normal }
\end{aligned}
$$

and

$$
\begin{aligned}
A C & =A B \cos \beta / \cos (\beta-\varphi), \\
& =\cos \beta / \cos (\beta-\varphi) \quad \text { with } \overline{A B} \text { as unit } .
\end{aligned}
$$

The energy flux is therefore:

$$
\varrho \omega^{2} G^{2} V_{0} \frac{\cos B}{\cos (\beta-\varphi)} \cdot \sin ^{2} \omega\left(\frac{x \sin \varphi+z \cos \varphi}{\bar{V}}-t\right) .
$$

Eq. [5] shows that the energy flux varies periodically. The net flux can be found by averaging over a period. 
One has further the following definition

$$
V_{s} \cos (\beta-\varphi)=V_{\varphi} .
$$

Eq. [5] becomes then (using [6]):

Energy $f u x=\varrho \omega^{2} G^{2} V^{2} \frac{\cos \beta}{\bar{V}_{\varphi}} \cdot \sin ^{2} \omega\left(\frac{x \sin \varphi+z \cos \varphi}{\bar{V}}-t\right)$.

Similarly the fluxes of the other waves can be determined:

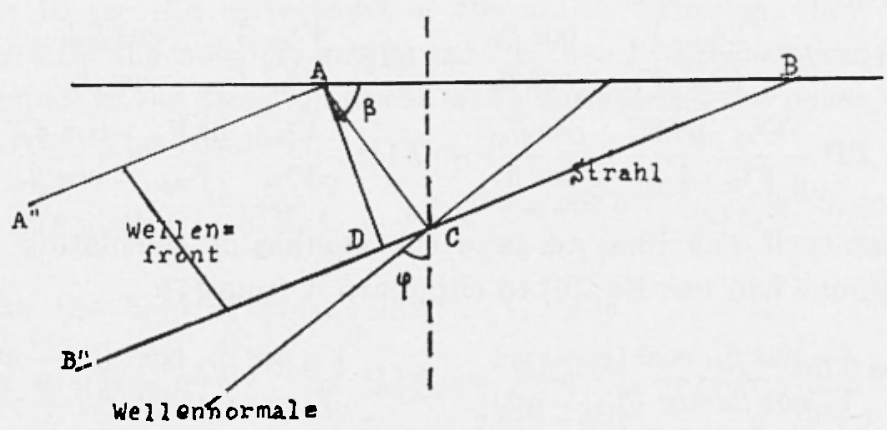

Fig. 1 - Energy considerations. Wellenfront $=$ wave front. Strahl $=$ ray. Wellennormale $=$ wave normal.

Reflected wave of I type:

$$
\left.F_{r 1}=\varrho \omega^{2} G_{r 1}^{2} V^{2}{ }_{s 1} \frac{\cos \beta_{r 1}}{\bar{V}_{r 1}} \cdot \sin ^{2} \omega: \frac{\left(x \sin \varphi^{*}-z \cos \phi^{*}\right.}{V_{r 1}}-t\right),
$$

Reflected wave of II type:

$$
F_{r 2}=\varrho \omega^{2} G_{r 2}^{2} V^{2}, \frac{\cos \beta_{r 2}}{V_{r 2}} \cdot \sin ^{2} \omega\left(\frac{x \sin \gamma-z \cos \gamma}{\bar{V}_{r 2}}-t\right),
$$

Refracted wave of I type:

$$
F_{d 1}=\varrho^{*} \omega^{2} G_{d 1}^{2} V_{s 3}^{2} \frac{\cos \beta_{d 1}}{V_{d 1}} \cdot \sin ^{2} \omega\left(\frac{x \sin \delta+z \cos \delta}{V_{d 1}}-t\right),
$$

Refracted wave of II type:

$$
F_{d 2}=\varrho^{*} \omega^{2} G_{d 2}^{2} V_{s 4}^{2} \frac{\cos \beta_{d 2}}{V_{d 2}} \cdot \sin ^{2} \omega\left(\frac{x \sin \eta+z \cos \eta}{V_{d 2}}-t\right)
$$

Here are $\bar{\rho}, \beta_{r 1}, \beta_{r 2}, \beta_{d 1}, \beta_{d 2}$ and $\varphi, \varphi^{*}, \gamma, \delta, \eta$ the angles of rays and wave normals of the incident, reflected wave of I and II type, refracted waves of I and II type respectively. The index $s$ in $V_{* 1}$ etc. points to the ray velocity (German: Strahlchgeswindigkeit). 
According to the law of the conservation of energy, the total energy of the derived waves must be equal to that of the incident:

$$
\begin{aligned}
& \varrho \omega^{2} G^{2} V^{2}{ }_{1 e} \frac{\cos \beta}{V_{e}}=g \omega^{2} G_{r 1}^{2} V_{s 1}^{2} \frac{\cos \beta_{r 1}}{V_{r 1}}+o \omega^{2} G_{r 2}^{2} V_{s 2}^{2} \frac{\cos \beta_{r 2}}{V_{r 2}}+ \\
& +\ldots o^{*} \omega-G_{d 1}^{2} V_{s 3}^{2} \frac{\cos \beta_{d 1}}{V_{d 1}}+o^{\cdots} \omega^{-\mathcal{U}^{2}{ }_{d 2} V^{2}{ }_{s 4}} \frac{\cos \beta_{d 2}}{Y_{d 2}}
\end{aligned}
$$

or with the incident flux as a unit:

$$
\begin{aligned}
& 1=R I^{2} \frac{V_{s 1}^{2}}{V_{s e}^{2}} \frac{V_{e}}{V_{r 1}} \cdot \frac{\cos \beta_{r 1}}{\cos \beta_{e}}+R I I^{2} \frac{V^{2}{ }^{2}}{V_{s e}^{2}} \frac{V_{e}}{V_{r 2}} \cdot \frac{\cos \beta_{r 2}}{\cos \beta_{e}}+ \\
& +T I^{2} \frac{\bar{V}_{s 3}^{2}}{\varrho V_{s e}^{2}} \frac{\varrho^{\tau} \bar{V}_{e}}{V_{d 1}}-\frac{\cos \beta_{d 1}}{\cos \beta_{e}}+T I I^{2} \frac{V_{s 4}^{2}}{\varrho V_{s e}^{2}} \frac{\varrho^{*} V_{e}}{V_{d_{2}}} \cdot \frac{\cos \beta_{d \varepsilon}}{\cos \beta_{e}} .
\end{aligned}
$$

Further if one likes to save the trouble of calculating the ray velocity, one can use Eq. [6] to eliminate it from [7]:

$$
\begin{aligned}
1= & R I^{2} \frac{V_{r 1} \cos \beta_{r 1} \cos ^{2}\left(\beta_{e}-\varphi\right)}{V_{e} \cos \phi_{e} \cos ^{2}\left(\beta_{r 1}-\varphi^{*}\right)}+n I I^{2} \frac{V_{r_{2}} \cos \beta_{r_{2}} \cos ^{2}\left(\beta_{c}-\varphi\right)}{V_{e} \cos \beta_{c} \cos ^{2}\left(\beta_{r 2}-\gamma\right)}+ \\
& +T I^{2} \frac{V_{d 1} \varrho^{r} \cos \bar{p}_{d 1} \cos ^{2}\left(\beta_{0}-\varphi\right)}{V_{e} \varrho \cos \beta_{e} \cos ^{2}\left(\beta_{d 1}-\delta\right)}+T I I^{2} \frac{V_{d 2} \varrho^{*} \cos \beta_{d e} \cos ^{2}\left(\beta_{2}-\psi\right)}{V_{e} \varrho \cos \beta_{e} \cos ^{2}\left(\beta_{d 2}-\eta\right)}
\end{aligned}
$$

with:

$R I=$ the reflexion coefficient (amplitudes) of wave of the I type, $R I I=$ the reflexion coefficient (amplitudes) of wave of II type, $T I=$ the transmission coefficient (amplitudes) of wave of I type, $T I I=$ the transmission coefficient (amplitudes) of wave of II type.

(Note that RI ...TII are to be determined as described in the last article) $\left({ }^{2}\right)$.

If in the medium $I$, that is the one containing the source of disturbance, the symmetry axis coincides with the normal to the boundary surface, $\beta_{c}$ can be set equal to $\beta_{r 1}$ or $\beta_{r_{2}}$ according as the incident wave is of I or II type.

[The astrisks wherever they occur, characterise the elements belonging to the medium II (not containing the source)].

For the wave of I type:

$$
\begin{gathered}
1=R I^{2}+R I I^{2} \frac{V_{r 2} \cos \beta_{r 2} \cos ^{2}\left(\beta_{c}-\varphi\right)}{V_{c} \cos \beta_{c} \cos ^{2}\left(\beta_{r_{2}}-\gamma\right)}+ \\
+T I^{2} \frac{V_{d 1} \cos \beta_{d 1} \cos ^{2}\left(\beta_{c}-\varphi\right) \varrho^{*}}{V_{c} \cos \beta_{c} \cos ^{2}\left(\beta_{d 1}-\delta\right) \varrho}+T I I^{2} \varrho^{* V_{d 2} \cos \bar{\delta}_{d 2} \cos ^{2}\left(\beta_{e}-\varphi\right)} \frac{V_{c} \cos \beta_{c} \cos ^{2}\left(\beta_{d 2}-\eta\right)}{\varrho}
\end{gathered}
$$


For the wave of II type:

$$
\begin{aligned}
1 & =R I^{2} \frac{V_{r 1} \cos \phi_{r 1} \cos ^{2}\left(\beta_{e}-\varphi\right)}{V_{e} \cos \beta_{e} \cos ^{2}\left(\beta_{r 1}-\varphi^{*}\right)}+ \\
& +R I I^{2}+T I^{2} \frac{\varrho^{*} V_{d 1} \cos \beta_{d 1} \cos ^{2}\left(\beta_{e}-\varphi\right)}{\varrho V_{e} \cos \beta_{e} \cos ^{2}\left(\beta_{d 1}-\delta\right)}+ \\
& -T I I^{2} \frac{\varrho^{*} V_{d 2} \cos \phi_{d 2} \cos ^{2}\left(\beta_{e}-\varphi\right)}{\varrho V_{e} \cos \beta_{e} \cos ^{2}\left(\beta_{d 2}-\eta\right)} .
\end{aligned}
$$

In order to see the correctness of the above formulae, they may be specialised for the isotropic media, that is, the ray direction and velocity are set equal to the direction of the wave normal and the phase velocity respectively. The result is:

$$
1=R I^{2}+R I I^{2} \frac{V_{r^{2}} \cos \gamma}{V_{e} \cos \varphi}+T I^{2} \frac{\varrho^{*} V_{d l} \cos \delta}{\varrho V_{e} \cos \varphi}+T I I^{2} \frac{\varrho^{* * V_{d 2}} \cos \eta}{\varrho V_{e} \cos \varphi},
$$

and with the help of Snell's law:

$$
1=R I^{2}+R I I^{2} \frac{\sin 2 \gamma}{\sin 2 \varphi}+T I^{2} \frac{\rho^{*} \sin 2 \delta}{\varrho \sin 2 \varphi}+T I I^{2} \frac{\varrho^{*} \sin 2 \eta}{\varrho \sin 2 \varphi} .
$$

This is the same expression as obtained by Blut $\left(^{6}\right)$ for isotropic media.

\section{Spectal CASES.}

In the following, the above formulae would be specialised for a few interesting cases, which occur frequently in nature.

\section{(1) Free Surface.}

Here the density and the velocities would practically vanish in medium II. Setting this in Eq. [7], [8] etc. one gets:

$$
1=R I^{2} \frac{\bar{V}_{s 1} \bar{V}_{e} \cos \beta_{r 1}}{V_{s e}^{2} V_{r 1} \cos \beta_{e}}+R I I^{2} \frac{V_{s 2}^{2} V_{e} \cos \beta_{r 2}}{V_{s e}^{2} V_{r 2} \cos \beta_{e}}
$$

(2) Incidence against a liquid.

The following changes would be made for an incident longitullinal wave:

$$
V_{s 3}=V_{d 1}, V_{s 4}=V_{d 2}=0, \beta_{d 1}=\delta,
$$

to get:

$1=R I^{2} \frac{V_{s 1}^{2} V_{e} \cos \beta_{r 1}}{V_{s e}^{2} V_{r 1} \cos \beta_{e}}+R I I^{2} \frac{V_{s 2}^{2} V_{e} \cos \beta_{r 2}}{V_{s e}^{2} V_{r 2} \cos \beta_{e}}+T I^{2} \frac{V_{d 1} V_{e} \cos \delta \varrho^{*}}{V_{s e}^{2} \cos \beta_{e} \varrho}\lfloor 11\rfloor$ 
and:

$$
\begin{gathered}
1=R I^{2} \frac{V_{r 1} \cos \beta_{r 1} \cos ^{2}\left(\beta_{e}-\varphi\right)}{V_{e} \cos \beta_{e} \cos ^{2}\left(\beta_{r 1}-\varphi^{*}\right)} \div \frac{n T^{2}}{V_{r 2} \cos \beta_{r 2} \cos ^{2}\left(\beta_{\varepsilon}-\varphi\right)} \\
+T I^{2} \frac{\varrho^{*} V_{d 1} \cos \delta \cos ^{2}\left(\beta_{e}-\varphi\right)}{\varrho V_{e} \cos \beta_{e}}+ \\
+
\end{gathered}
$$

(3) If the medium I be liquid, then the following substitutions are to be made,

$$
\beta_{r 1}=\hat{\mu}_{\bullet}, V_{s 2}=V_{r_{2}}=0, V_{s 1}=V_{r 1}=V_{s s}=V_{e},
$$

to obtain:

$$
1=R I^{2}+T I^{2} \frac{\varrho^{*} V^{2}{ }_{s 3} V_{e} \cos \beta_{d 1}}{\varrho V^{2} V_{d 1} \cos \varphi}+T I I^{2} \frac{\stackrel{n}{*}^{*} V^{2}: \therefore}{\varrho V_{e} V_{d 2} \cos \varphi},
$$

and

$1=R I^{2}+T I^{2}-\frac{\varrho^{*} V_{d 1} \cos \beta_{d 1}}{\varrho V_{e} \cos \varphi \cos ^{2}\left(\beta_{d 1}-\delta\right)}+T I I^{2} \frac{\varrho^{*} V_{d 1} \cos \beta_{d^{2}}}{\varrho V_{e} \cos \varphi \cos ^{2}\left(\beta_{d_{2}}-\eta\right)}$.

(4) One of the media: solid isotropic.

(a) Let the medium I be isotropic solid. With the coincidence of the ray direction with the wave normal, one gets for the incident $P$-wave:

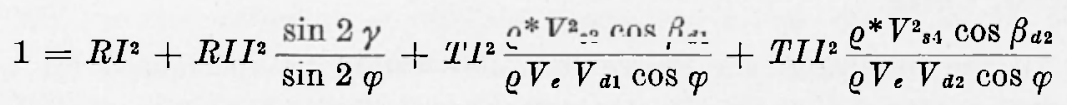

and for incident $S$-wave:

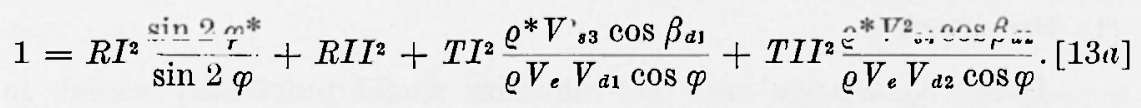

(b) If the medium II be isotropic solid, one would get for the incident $P$-wave:

$$
\begin{aligned}
1 & =R I^{2} \frac{V_{r 1} \cos \beta_{r 1} \cos ^{2}\left(\beta_{e}-\varphi\right)}{V_{e} \cos \beta_{e} \cos ^{2}\left(\beta_{r 1}-\varphi^{*}\right)}+R I I^{2} \frac{V_{r 2} \cos \beta_{r 2} \cos ^{2}\left(\beta_{e}-\varphi\right)}{V_{e} \cos \beta_{e} \cos ^{2}\left(\beta_{r 2}-\gamma\right)}-+ \\
& +T I^{2} \frac{\varrho^{*} V_{d 1} \cos \delta \cos ^{2}\left(\beta_{e}-\varphi\right)}{\varrho V_{e} \cos \beta_{e}}+T I I^{2} \frac{\varrho^{*} V_{d 2} \cos \eta \cos ^{2}\left(\beta_{e}-\varphi\right)}{\varrho V_{e} \cos \beta_{e}}
\end{aligned}
$$

There would be more simplifications in the formulae, if the symmetry axes coincide with the normal 'to the boundary surface, as then the angle of reflexion of the $P(S)$ waves would be equal to that of the incident $P(S)$ waves. 
The energy flux: Calculation from the POTENTIAL ENERgy.

As mentioned above the energy flux can be calculated from Eq. [2]. For the energy ratios one needs to consider only the normal component of the energy flux.

For the transversely isotropic media, the elastic potential function is given by $\left({ }^{4}\right)$ ):

$$
\begin{aligned}
2 W & =A\left(e_{x x}^{2}+e_{y y}^{2}\right)+C e_{z z}^{2}+2 F\left(e_{x x}+e_{y y}\right) e_{z z}+ \\
& +2(A-2 N) e_{x x} e_{y y}+L\left(e_{y z}^{2}+\hat{e}_{z x}\right)+N e_{x y}^{2} .
\end{aligned}
$$

The stress tensor needed is further:

$$
\tau_{i k}=\frac{\partial W}{\partial e_{i k}}
$$

The equations [15], [15a], and [2] yield:

(a) Arbitrary Orientation of the Symmetry Axes;

Incident wave of I or II type:

$$
F_{z}=\frac{\omega^{2} G^{2} e}{2 V N^{2}(\alpha)}\left[g _ { k } ( \alpha , \varphi ) \left(\overline{p \sin ^{2} \alpha+q_{k} \cos \alpha \sin 2 \varphi}+\right.\right.
$$

$\left.+2 L r_{\alpha k k} \sin \alpha \cos 2 \varphi\right)+2 f_{k}(\alpha, \varphi) \cos \alpha\left(\overline{p \sin ^{2} \alpha \sin ^{2} \varphi+s \sin ^{2} \alpha-}\right.$

$\left.\left.-q_{k} \sin ^{2} \varphi+L r_{\sigma k} \sin \alpha \sin 2 \varphi\right)+2 C\left(\varrho V_{k}^{2}-L\right)^{2}\right] \sin ^{2} \omega=$

$$
=\frac{\omega^{2} G_{e}^{2}}{2 V_{k} N^{2}(\alpha)} R_{k}(\alpha, \varphi) \sin ^{2}\left(\frac{x \sin \alpha+z \cos \alpha}{V}-t\right) \text {. }
$$

With the introduction of the following abbreviations:

$$
\begin{array}{ll}
p & =(L+F)(A-F)+(A-L)(C-F), \\
q_{k} & =(C-F)\left(L-\varrho V_{k}^{2}\right), \\
r_{\alpha k} & =F+\varrho V_{k}^{2}-(A+F) \sin ^{2} \alpha \\
s & F(L+F)-C(A-L), \\
g_{k}(\alpha, \varphi)= & (L+F) \sin 2 \alpha \cos \varphi+2\left(\overline{A-L} \sin ^{2} \alpha+L-\varrho V_{k}^{2}\right) \sin \varphi, \\
f_{k}(\alpha, \varphi)= & (L+F) \sin \alpha \cos \alpha \sin \varphi+\left(L-A \sin ^{2} \alpha-L+\varrho V_{k}^{2}\right) \cos \varphi, \\
R_{k}(\alpha, \varphi)= & \text { the expression in the brackets, with } k=1,2 \text { according } \\
& \text { to the wave type. }
\end{array}
$$


The energy flux normal to the boundary surface must be continuous, otherwise the energy density would change there enormously. One would get then:

$$
\begin{aligned}
1 & =R^{2} \frac{V R_{1}(\beta, \varphi) N^{2}(\alpha)}{V_{1} R(\alpha, \varphi) N^{2}(\beta)}+R I I^{2} \frac{V R_{2}(\gamma, \varphi) N^{2}(\alpha)}{V_{2} R(\alpha, \varphi) N^{2}(\beta)}+ \\
& +T^{2} \frac{V R_{1}^{*}\left(\delta, \varphi^{*}\right) N^{2}(\alpha)}{V_{1} R(\alpha, \varphi) N^{* 2}(\delta)} \div \stackrel{\because}{-}-\frac{V R_{2}^{*}\left(\eta, \varphi^{*}\right) N^{2}(\alpha)}{V_{2}^{*} R(\alpha, \varphi) N^{* 2}(\eta)}
\end{aligned}
$$

with the incident flux as unit.

\section{Special Cases.}

The above equations would be simplified to some extent, if they are specialised for some cases, which occur in nature. As before the following specialisations may be suggested:

\section{(1) Free Surface.}

In this case all terms containing the transmission coefficients vanish identically in Eq. [18]. That is, one can set $T I$ and $T I I$ equal to zero there to get the required expression. In other words [18] would not consist of its second row here.

\section{(2) Incidence against a liquid.}

Since the liquids can be considered to be isotropic media where $P$-waves only can exist, one would have:

$$
R_{1}^{*}\left(\gamma, \varphi^{*}\right)=2 \lambda^{* 3} \cos ^{2} \gamma, \quad N^{* 2}(\gamma)=\lambda^{* 2} \cos ^{2} \gamma,
$$

and thus the energy equation:

$$
\begin{gathered}
1=R I^{2} \frac{V R_{1}(\beta, \varphi) N^{2}(\alpha)}{V_{1} R(\alpha, \varphi) N^{2}(\beta)}+R I I^{2} \frac{V R_{-i}(\hat{\gamma}, r) N^{2}(\alpha)}{V_{2} R(\alpha, \varphi) N^{2}(\gamma)}-1 \\
+T I^{2} \frac{2 N^{2}(\alpha) V^{*} V \varrho^{*}}{R(\alpha, \varphi)}
\end{gathered}
$$

where $V^{*}$ is the $P$-wave velocity in the liquid. If in the solid medium the symmetry plane coincides with the surface of discontinuity, $\alpha=\beta$ and $\varphi=0$. Then the above equation becomes:

$$
1=R I^{2}+R I I^{2} \frac{V R_{2}(\gamma, 0) N^{2}(\alpha)}{V_{2} R(\alpha, 0) N^{2}(\gamma)}+T I^{2} \frac{V N^{2}(\alpha) \rho^{* 2} V^{*}}{R(\alpha, 0)}
$$


with

$$
\begin{aligned}
R_{k}(\alpha, 0) & =(L+F) \sin ^{2} \alpha \cos \alpha L r_{\alpha k}+C\left(L-\varrho V^{2}\right)^{2}- \\
& -s \cos \alpha \sin ^{2} \alpha\left(A-L \sin ^{2} \alpha+L-\varrho V^{2}\right)= \\
& =-\cos \alpha\left(\alpha \sin ^{4} \alpha+\beta \sin ^{2} \alpha+\gamma\right)= \\
& =-\cos \alpha P(\alpha),
\end{aligned}
$$

with

$$
\begin{aligned}
& \overline{\overline{\alpha_{k}}}=A(L+F)^{2}-C(A-L)^{2}, \\
& \overline{\beta_{k}}=C\left(\varrho V_{k}^{2}-L\right) 2(A-L)-\varrho V_{k}^{2}(L+F)^{2}, \\
& \overline{\gamma_{k}}=-C\left(L-\varrho V_{k}^{2}\right)^{2}, \\
& P_{k}(\varphi)=\bar{\alpha} \sin ^{4} \varphi+\bar{\beta} \sin ^{2} \varphi+\overline{\bar{\gamma}} .
\end{aligned}
$$

Now if the medium containing the source is liquid, the energy equation would be obtained as:

$$
1=R I^{2}+T I^{2} \frac{R^{*}\left(\delta, r^{*}\right)}{2 \bar{V}_{1}^{*} \bar{N}^{* 2}(\delta) \varrho \bar{V}}+T I I^{2} \frac{R_{2}^{*}\left(\eta, \tilde{\psi}^{*}\right)}{2 V_{2}^{*} N^{* 2}(\eta) \varrho \bar{V}} .
$$

(3) There is a simple case, where the symmetry axes in both of the media coincide with the normal to the boundary surface. In this case one has simply to put $\varphi=\varphi^{*}=0$, to get:

$$
\begin{aligned}
1=R I^{2}+R I I^{2} & \frac{V_{e} N^{2}(\alpha) \cos \gamma P_{2}(\gamma)}{V_{2} N^{2}(\gamma) \cos \alpha P(\alpha)}-T I^{2} \frac{V_{e} N^{2}(\alpha) \cos \delta P_{1}^{*}(\delta)}{V_{*}^{*} N^{* 2}(\delta) \cos \alpha P(\alpha)}+ \\
& +T_{I} I^{*} \frac{V_{e} N^{2}(\alpha) \cos \eta P_{2}^{*}(\eta)}{V_{2}^{*} N^{* 2}(\eta) \cos \alpha P(\alpha)}
\end{aligned}
$$

with the incident energy flux along the normal to the boundary surface as a unit.

\section{(4) Isotropic solid medium $I$.}

In the present case $\varphi=0$. Further one has to account for the transition, of the anisotropy to the isotropy, that is:

$$
A=C=\lambda+2 \mu, F=\lambda \text {, and } L=\mu \text {. }
$$

The source would radiate only pure longitudinal or transversal waves. For the incident longitudinal wave one would get for example:

$$
\begin{aligned}
1=R I I^{2}+ & R I^{2} \frac{\sin 2 \underset{r}{\beta}}{\sin 2 \alpha}+T I^{2} \frac{R_{1}^{*}\left(\delta, \varphi^{*}\right)}{2 V_{1}^{*} N^{* 2}(\delta) \varrho V \cos \alpha}+ \\
& +T I I^{2} \frac{R_{2}^{*}\left(\eta, \hat{\psi}^{* !}\right)}{2 V_{i}^{*} N^{* 2}(\eta) \varrho V \cos \alpha} .
\end{aligned}
$$


The case of the incident transversal wave can also be treated similarly. One can check for himself that all the above equations lead to the same equation [9], after specialisation to isotropy.

In the above considerations it has been assumed that the angle of incidence does not exceed the smallest of the critical angles; there would exist at the most two such angles for the incident wave of the I type, and three for the II type. Therefore the time, $x$ - and $z-$ factors go into the expressions as:

$$
\sin ^{2} \omega\left(\frac{x \sin \alpha+z \cos \alpha}{V(\alpha)}-t\right)
$$

This shows that the direction of the energy flux and not its magnitude remains constant always over the whole boundary surface.

If the angle of incidence exceeds the smallest of the critical angles, it happens that depending upon the elastic constants at first the sine of the refracted angle becomes greater than unity and then after a certain incidence angle it becomes imaginary. In such a case the reflexion and transmission coefficients (amplitudes) $R I, \quad R I I, T I$ and $T I I$ become complex. The factor containing the time and space turns into

$$
\sin \omega\left(\frac{x \sin \alpha}{\nabla(\alpha)}-t+\varepsilon\right) \cos \omega\left(\frac{x \sin \alpha}{V(\alpha)}-t+\varepsilon\right) \exp (-z \cos \alpha / V)
$$

where $\varepsilon$ is the phase difference.

This shows quite clearly that the energy of such a wave is bound with the boundary surface; it decays exponentially with the distance from the surface. The direction of the flux depends upon the time as well as the position on the surface. At a given place it changes its sign every quarter of a period and for a given time it changes its sign every quarter of the wave length along the boundary surface. If the energy flux is integrated over a full period, it would vanish identically. The behaviour of the energy flux in anisotropic media has been investigated by Synge ( $\left.{ }^{5}\right)$ and Ossipor ( ${ }^{7}$ ). Tooley et al. $\left(^{8}\right)$ come to the conclusion, while investigating the same for the isotropic media, that the Knott's equations guarantee the continuity of the net and momentory energy flux within the smallest critical angle, but if it is exceeded, it is necessary that the imaginary and the real parts of the equation must be fulfilled for the continuity of the momentory flux. The same can be extended to the anisotropic media. 
The Calculated Results.

In the following figures (2-12) the energy ratios of the different derived waves are presented. The incident wave is of I type, that is the quasilongitudinal wave. They are represented as functions of the ray direction and the wave normals. The tables are not reproduced here due to their huge volume. The energy ratios for the same media as in the last article have been calcultated. They are also a very useful check on the correctness of the formulae derived above for the amplitude and energy ratios. The energy ratios of the derived waves add together to unity with an accuracy up to the ninth decimal place. The results were obtained through the computer Telefunken TR 4, which produces results up to the tenth decimal place with the rounding off error in the last.

Fig. 2 shows the energy ratios of zinc/beryl, along with three hypothetical isotropic media, having some of the elastic constants common with those of zinc and beryl. These isotropic media have the following elastic constants:

$$
\begin{aligned}
& \text { case 1: } A=C=14.3, L=4.0(A=C=\lambda+2 \mu, L=\mu, F=\lambda) \\
& A^{*}=C^{*}=23.63, L^{*}=6.53 \\
& \text { case 2: } A=C=14.3, L=5.5 \\
& A^{*}=C^{*}=26.94, L^{*}=10.165 \\
& \text { case 3: } A=C=5.0, L=0.85 \\
& A^{*}=C^{*}=23.63, L^{*}=8.51 \\
& \text { zinc: } A=14.3, C=5.0, F=3.3, L=4.0 \\
& \text { beryl: } A=26.94, C=23.63, F=6.61, L=6.53
\end{aligned}
$$

(the numbers on the curves in the figure refer to these cases).

The densities in the I and II medium were always the same, that is 7.1 and $2.7 \mathrm{gr} / \mathrm{cm}^{2}$ respectively. All the constants are in $10^{11} \mathrm{dynes} / \mathrm{cm}^{2}$. 
From the figure it can be seen clearly, what a difference the deviation from the isotropy in the media produces on the energy distribution in the derived waves: a maximum in $T 2$ is not present at all in the isotropic media.

Fig. 3 represents ice against schists. It shows that the refracted wave of I type is the major carrier of energy as in the isotropy. In Fig. 4 is represented a media-combination, where no critical angle exists. It can be seen that here also the refracted wave of I type carries the major part of the incident energy. It demonstrates further that in the range of $35^{\circ}-45^{\circ}$ (wave normals) or $40^{\circ}-60^{\circ}$ (ray direction) the reflected wave of I type and the refracted wave of II type are almost non-existent. This means that in the overlying medium (I) the reflected wave of II type only can be recorded in the said range of the incident angles. A very similar case is reproduced in Fig. 5, where the critical angle is $90^{\circ}$. Fig. 6 displays an interesting case, where medium $I$ is isotrope with the elastic constants approximating those of the upper crust, while the underlying medium is anisotrope, where the velocity of the $P$-waves in the horizontal direction is about $13 \%$ higher than that in the vertical. It can be seen from the figure, that only near the critical angle (about $64^{\circ}$ ) some of the energy is sent back. Fig. 7 represents media, which are anisotrope owing to the alternate layering of the sandstones and limestones $\left(^{\theta}\right)$. Here the energy in the refracted wave of II type is so small that it is not drawn in the figure.

In the last five diagrams is shown the influence of varying the different elastic parameters $A^{*}, \varrho^{*} / \varrho, C^{*} / C, F^{*} / F$, and $L^{*} / L$ on the energy distribution. It is worthwhile to notice that the critical angle changes only with $A^{*}$ and the density ratio.

For the time being the results have been presented for the incident wave of I type, up to the smallest critical angle. It is intended to extend them to all the angles (as well as to the wave of II type), owing to their significance for the wide angle reflexions. 

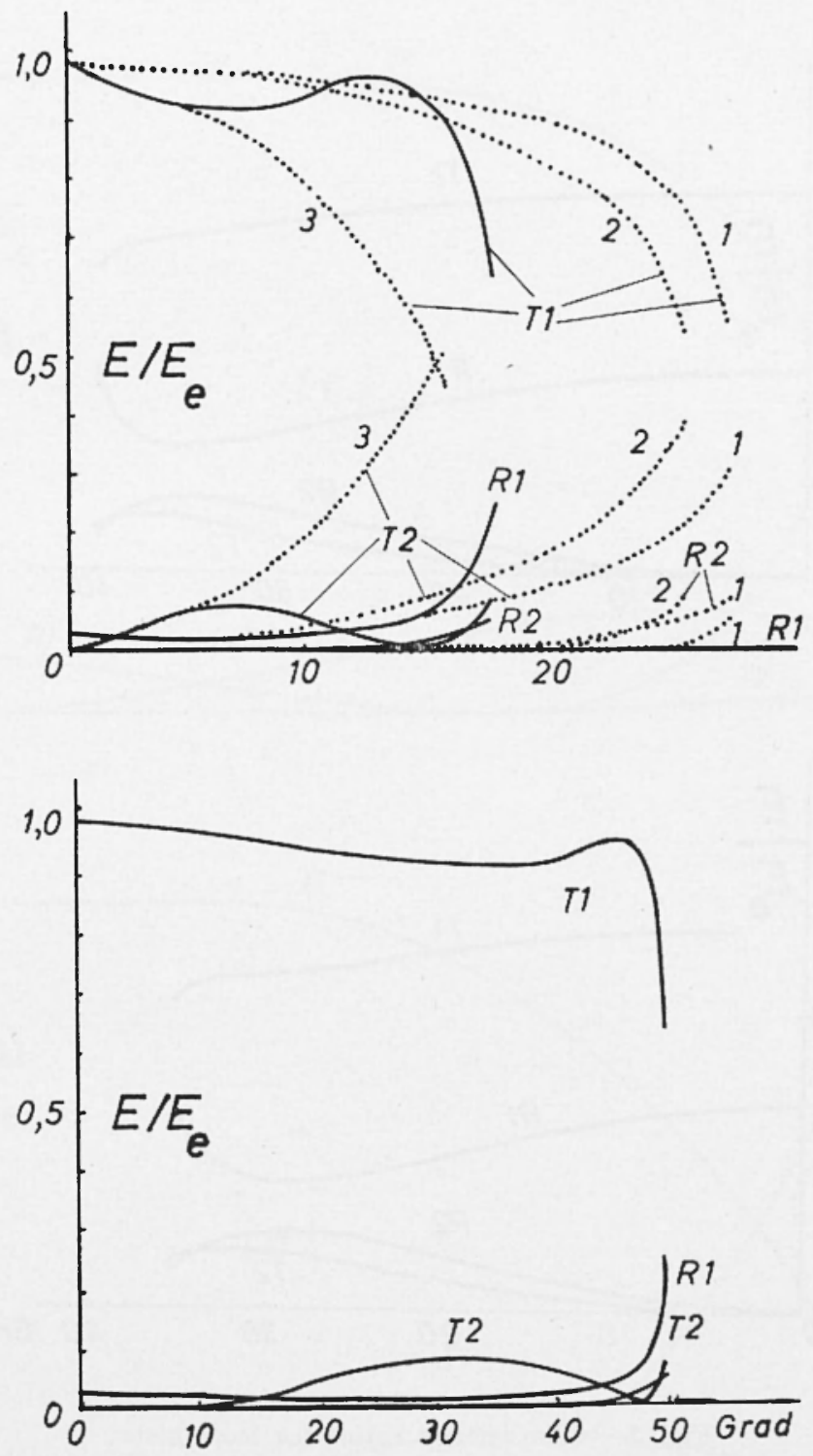

Fig. 2 - Energy ratios as function of wave normal direction (above) and ray direction (below) for zinc/beryl. The dotted curves are for the isotropic media. For explanation see text. 


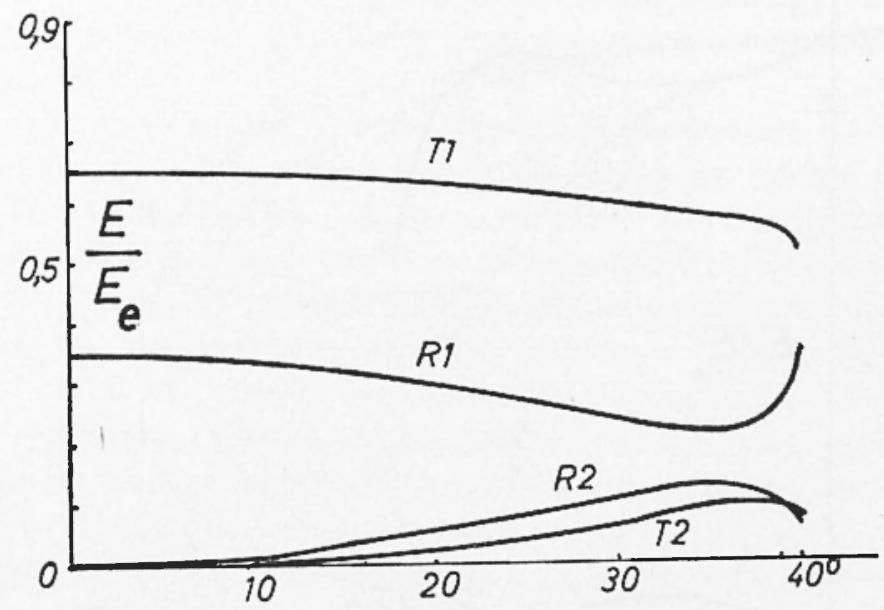

(c)

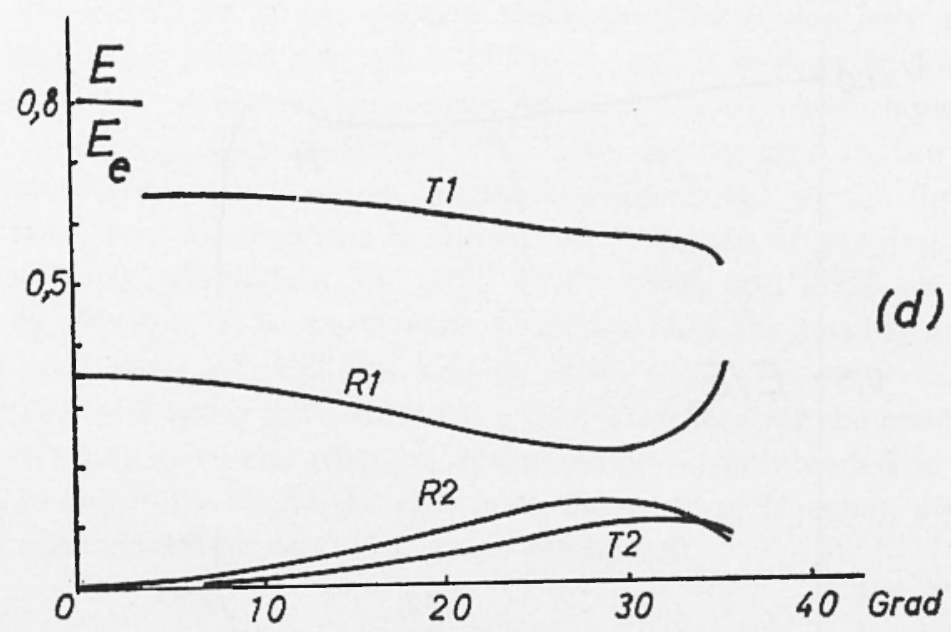

Fig. 3 - The energy ratios for ice/schists.

Ice: $\varrho=0.917, A=1.36, C=1.46, F=0.52, L=0.32$ in $10^{11} \mathrm{dynes} / \mathrm{cm}^{2}$ Schists: $\varrho=2.74, A=9,06, C=7,41, F=2,39, L=2.21$ " " 

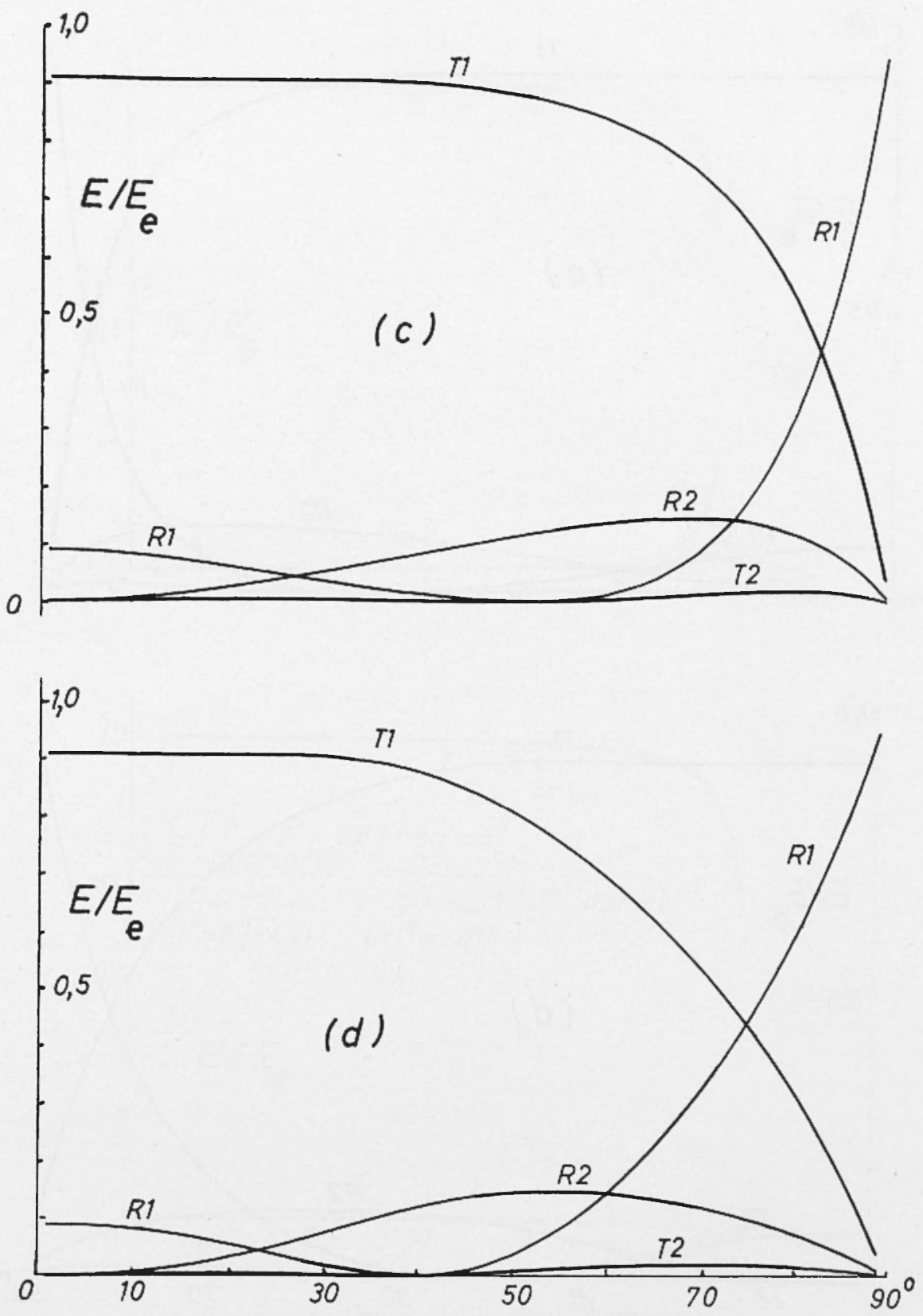

Fig. 4 - Energy ratios for: $-\varrho=2.64, \varrho^{*}=3.014, A=4, A^{*}=2, C=2.71$, $O^{*}=8.15, F=1.07, F^{*}=2.63, L=0.81, L^{*}=2.43$ in $10^{11}$ dynes $/ \mathrm{cm}^{2}$. 



Fig. 5 - Energy ratios. The constants are the same as in Fig. 4, except that $A^{*}=4$. 

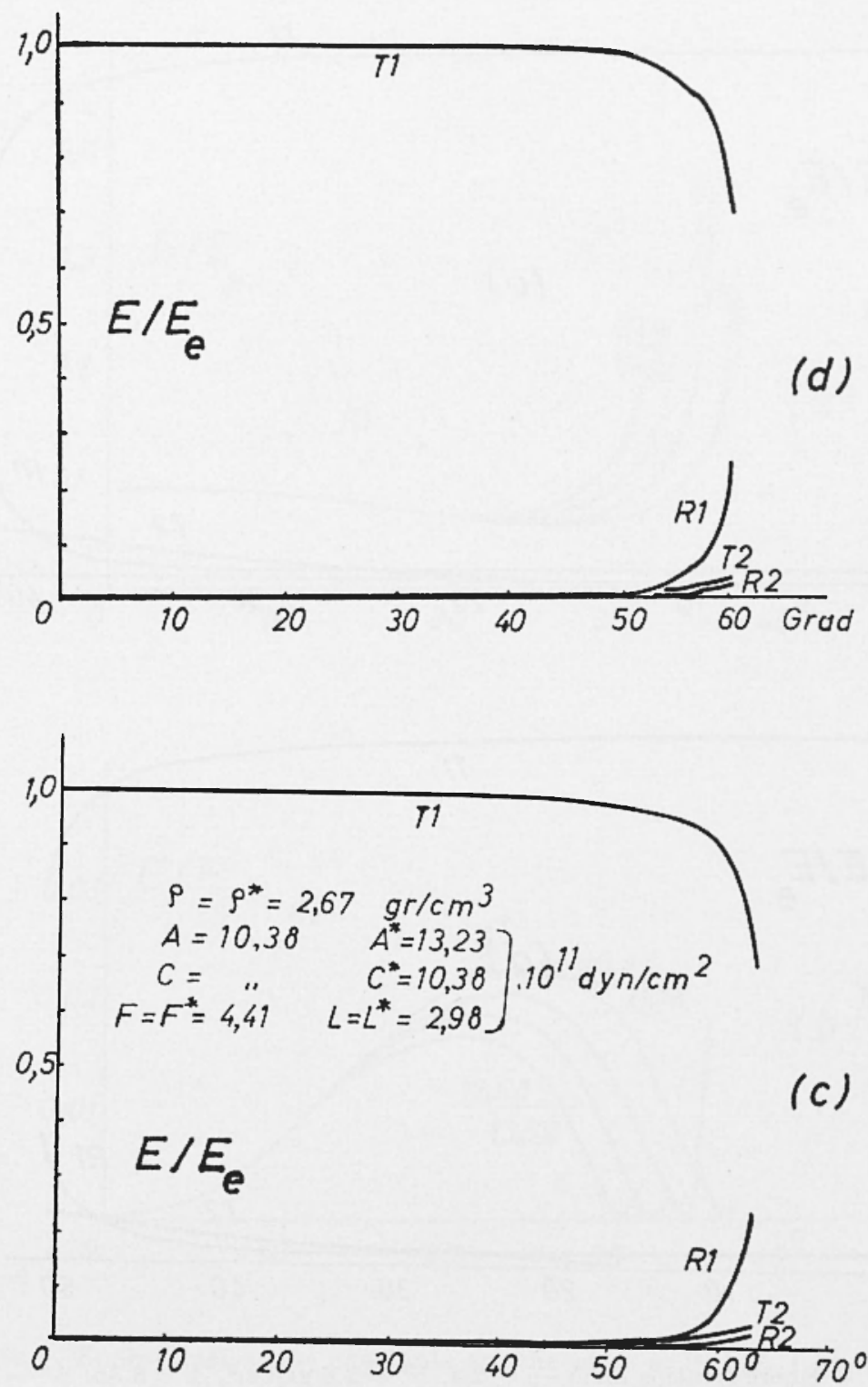

Fig. 6 - Energy ratios for medium I, being isotrope. 

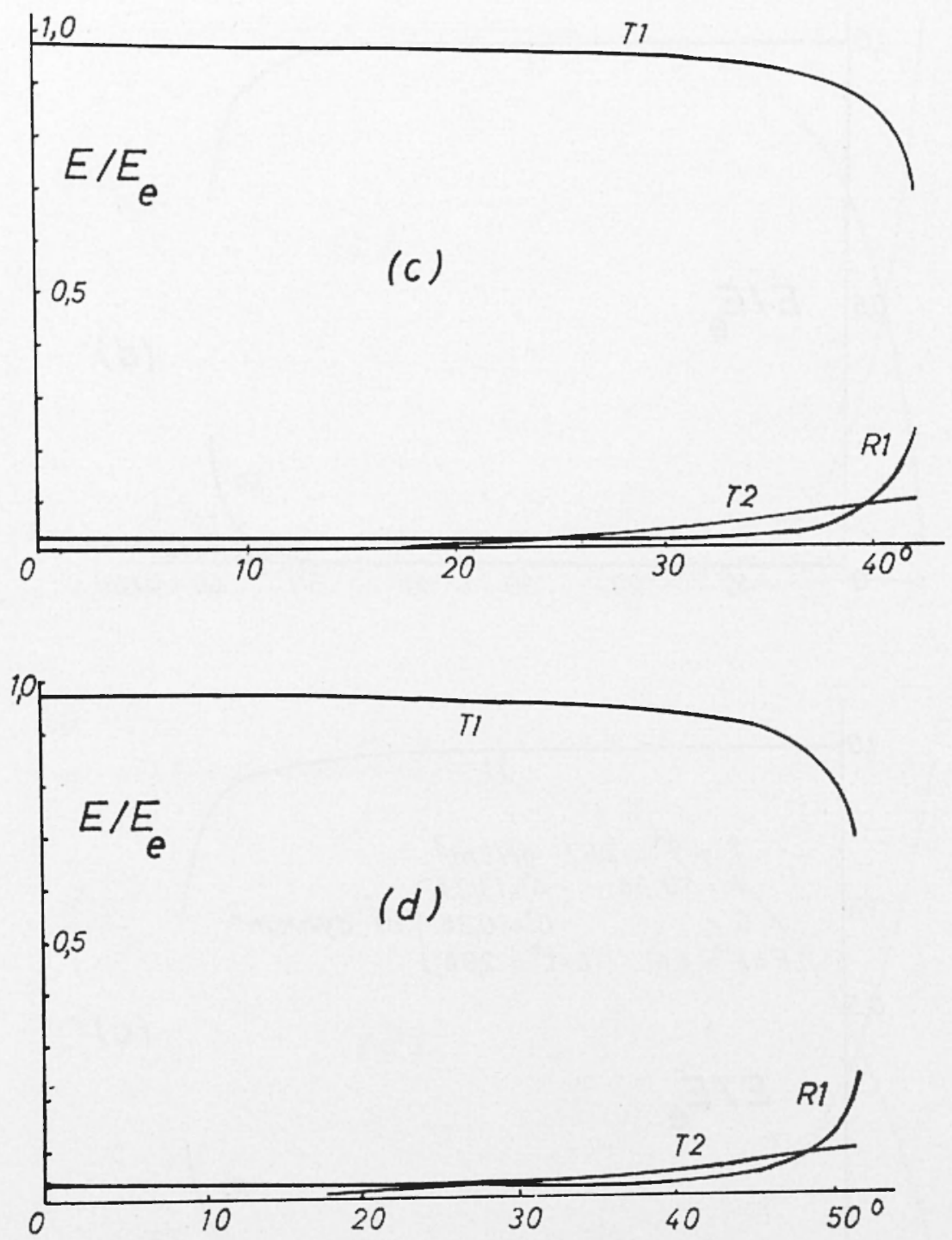

Fig. 7 - Energy ratios for: $-\varrho=2.4, O^{*}=2.6 \mathrm{gr} / \mathrm{cm}^{3}, A=3.36, A^{*}=6.25$, $C=2.46, C^{*}=4.57, F=0.97, F^{*}=1.74, L=0.74, L^{*}=1.40$ in $10^{11} \mathrm{dynes} / \mathrm{cm}^{2}$ $T 2$ is not drawn due to its negligible energy ratio. 

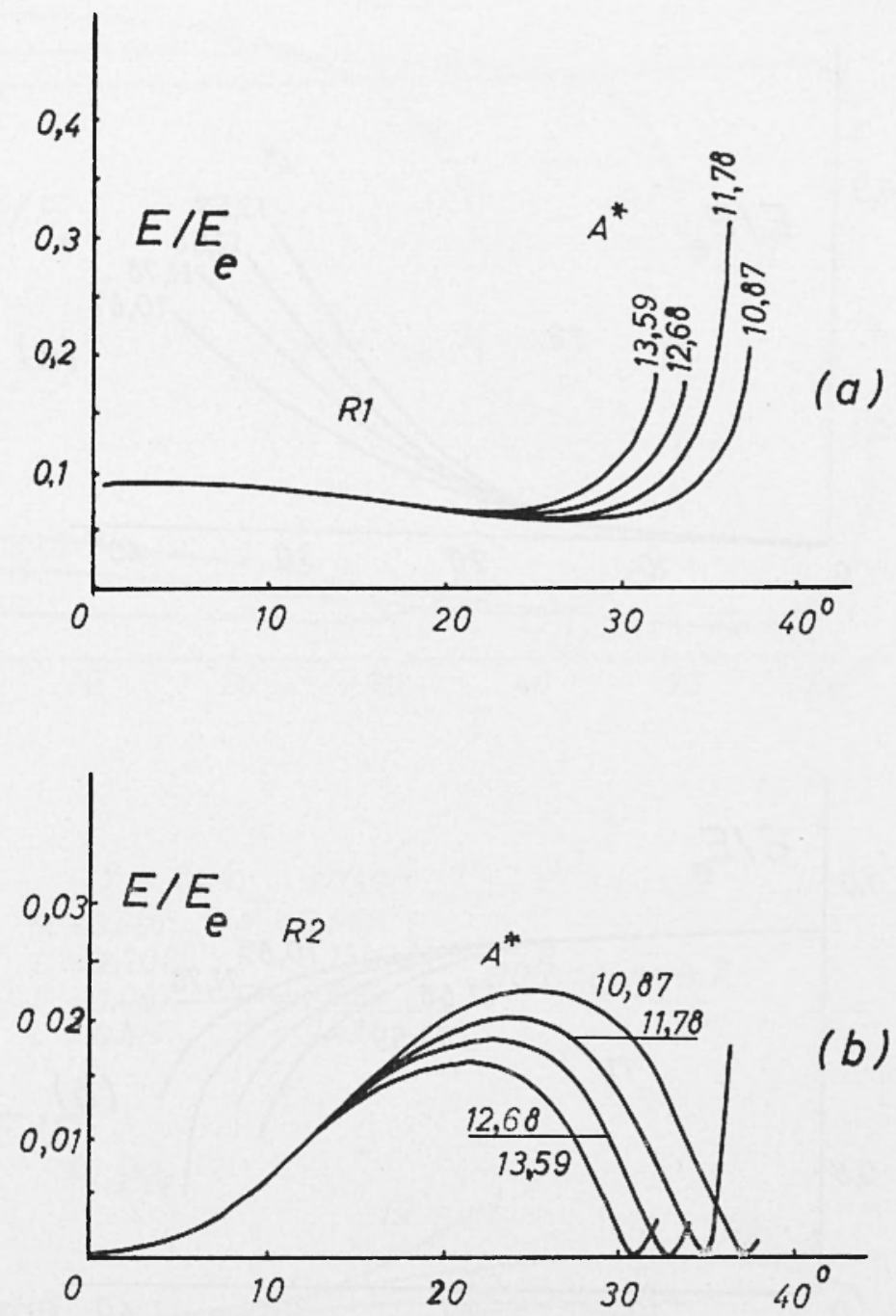

Fig. $8 a$ - Energy ratios: the constants are the same as in Fig. 4, excepting $A^{*}$, which is shown on the curves. 

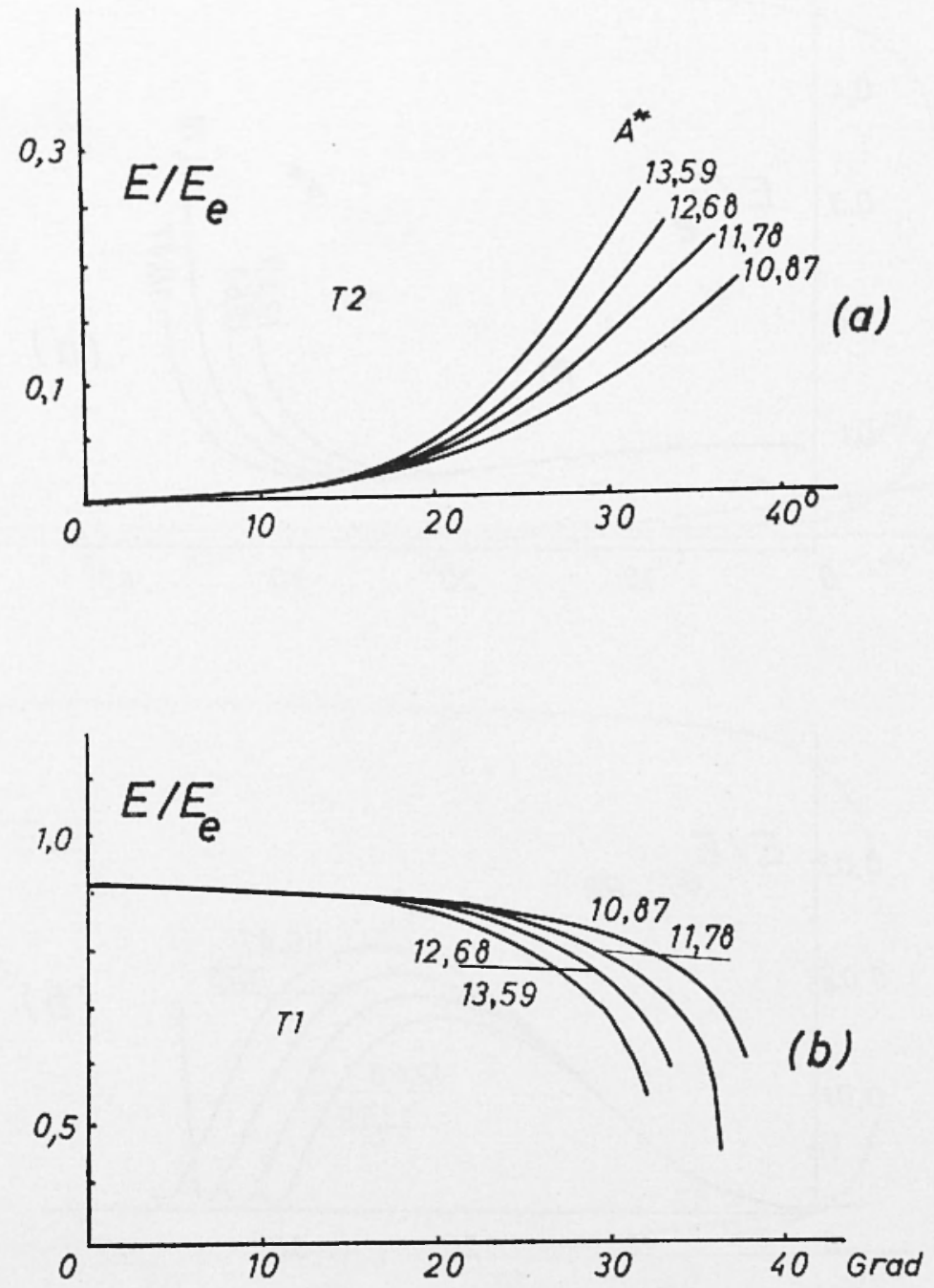

Fig. $8 b$ - The energy ratios (for text, see Fig. 8a). 

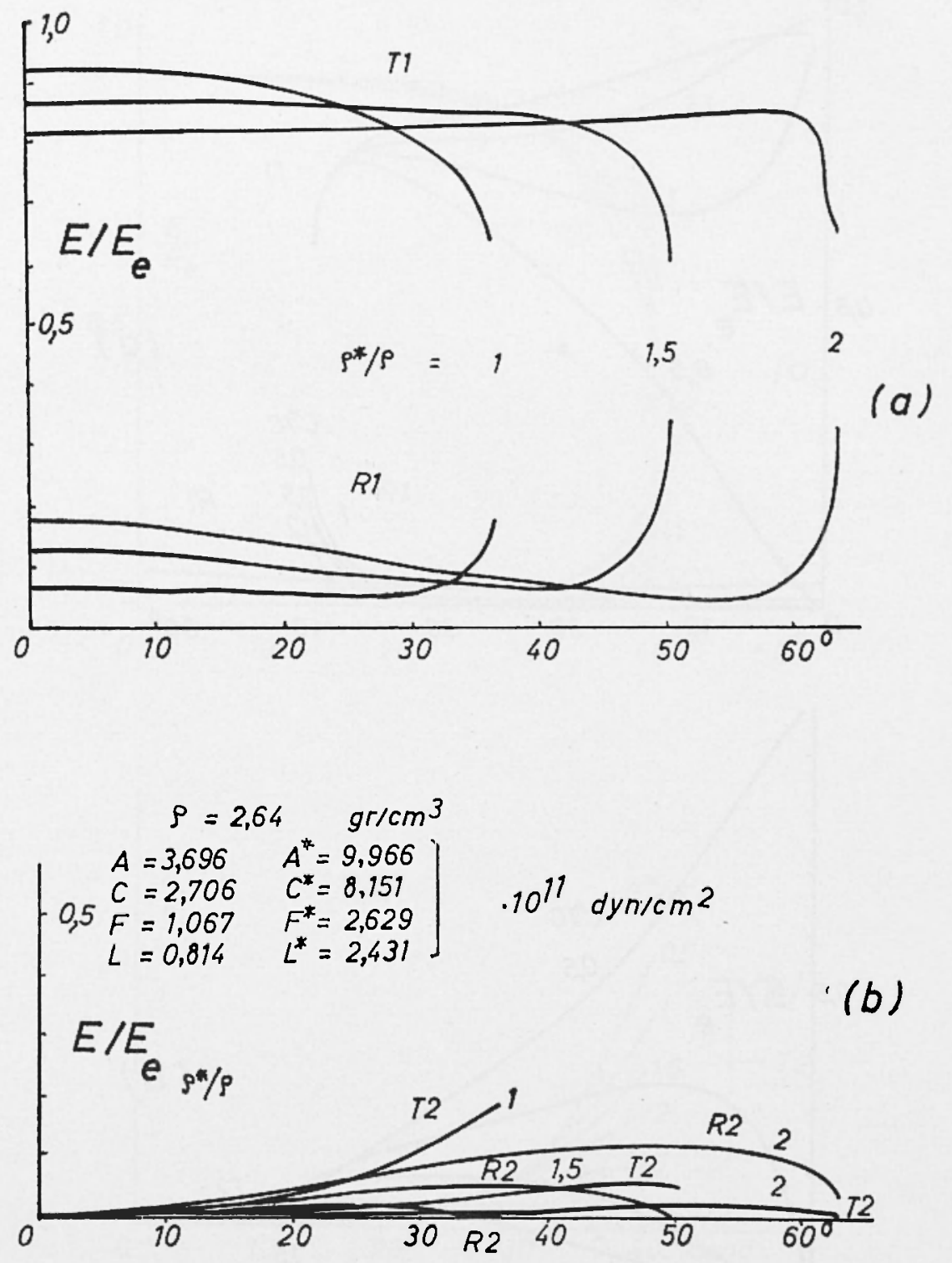

Fig. 9 - The energy ratios.

Influence of varying the density ratio $\varrho^{*} / \varrho$, shown on the curves. 

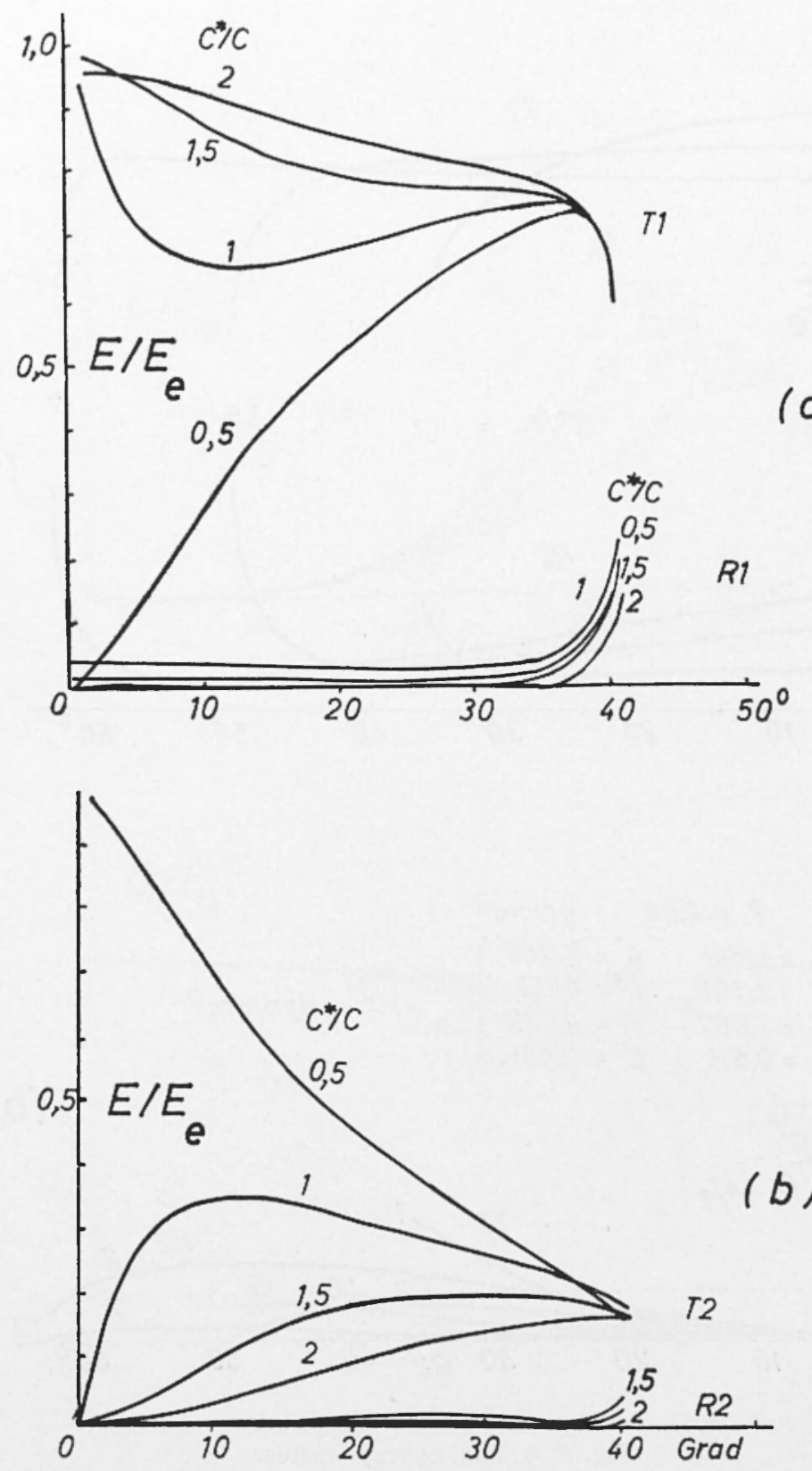

Fig. 10 - Energy ratios. Influence of varying the ratio $C^{*} / C$. For the other constants see Fig. 9. 

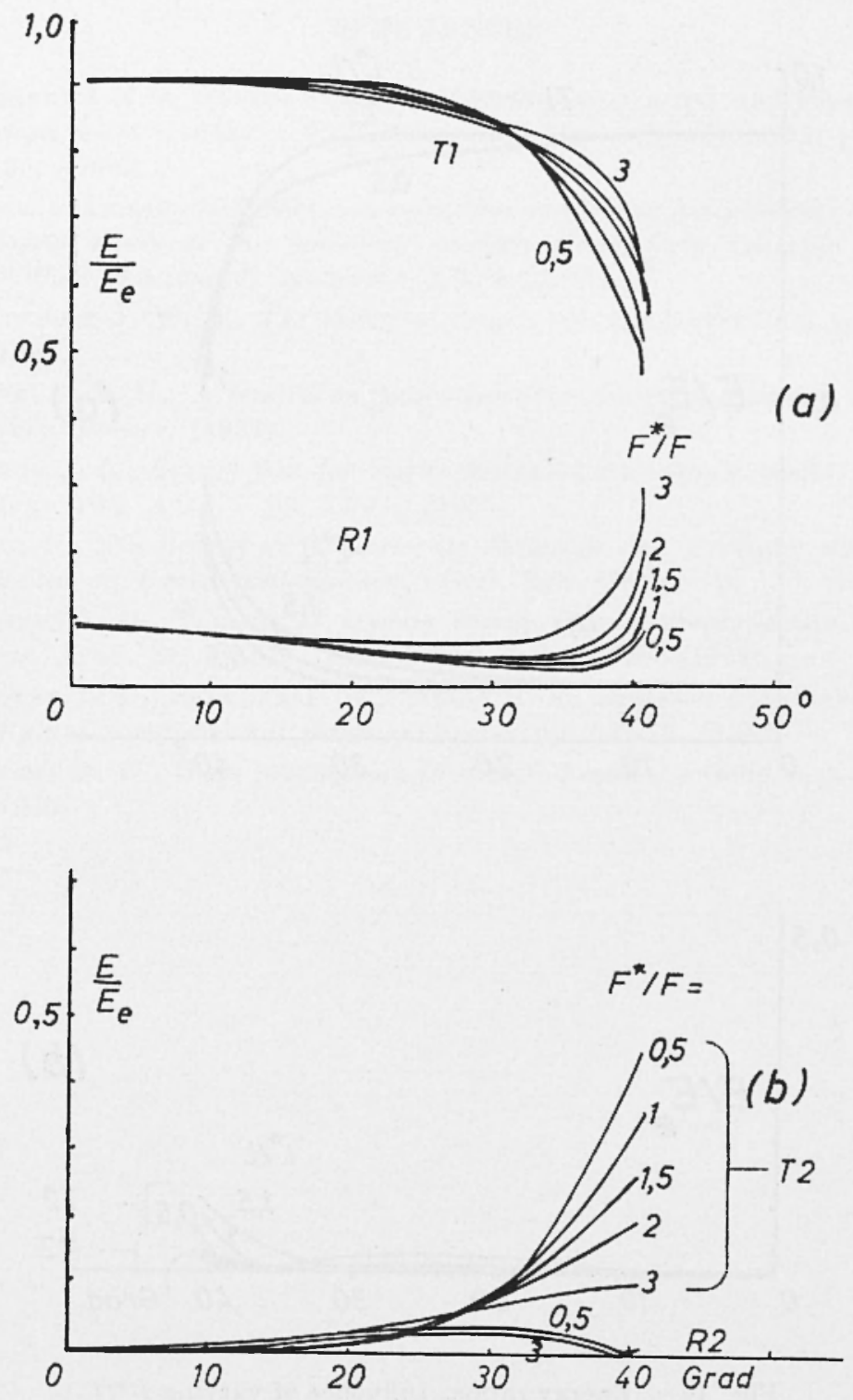

Fig. 11 - Energy ratios. Influence of varying $F^{*} / F$. For the other constants see Fig. 9. 

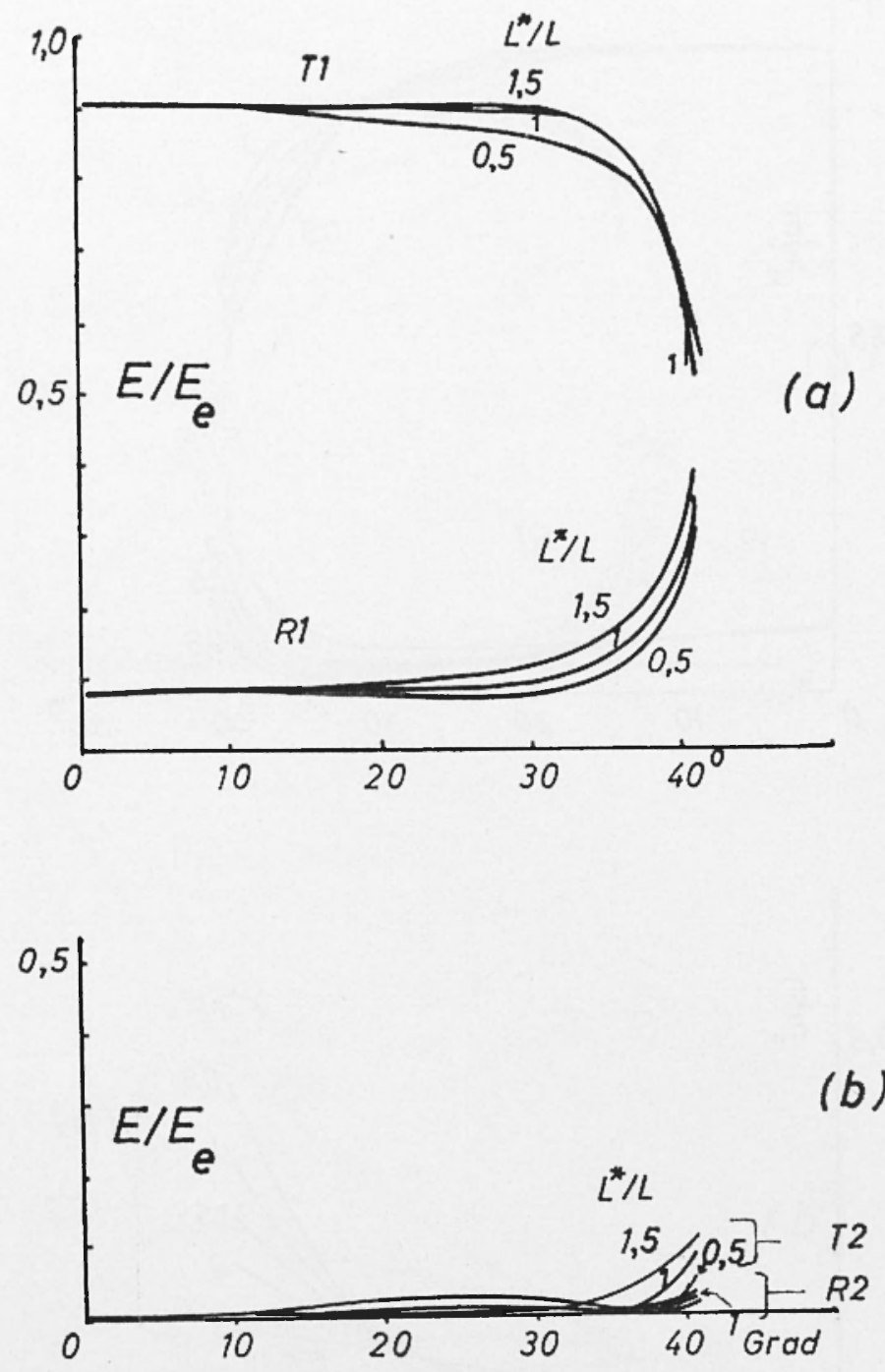

Fig. 12 - Energy ratios. Influence of varying $L^{*} / L$. For the other constants see Fig. 9. 


\section{REFERENCES}

(1) Lighthill M. J., Studies on magnetohydrodynamic waves and other anisotropic wave motions. "Phil. Tran. Roy. Soc." (Lond) A252, pp. 397. 430, (1906).

(2) Gui.zar Ahmad, Reflexion and refraction coefficients (amplitudes) of plane elastic waves at the boundary between transversely isotropic media, Ist Part. "Annali di Geofisica ", XX, 3, (1967).

(3) Rayleigir J. W. S., The theory of sound. vol. 1. "Dover Publ. n, p. 479, (1894.)

(4) Love A. E. H., A treatise on the mathematical theory of elasticity. "Camb. Univ. Press ", (1927).

(5) Srnge J. L., Energy flux for elastic waves in anisotropic media. "Proc. Roy. Irish Acad." pp. 13-21, (1956).

(6) Blut H., Ein Beitrag zur Theorie der Reflexion und Brechung elastischer Wellen an Unsteligkeitsflaechen. "Gerl. Beit. Geop.", p. 130, (1932).

(") Ossipov I. O., Transfer of seismic energy in anisotropic media. "Bull. Izw. Acad. Sc. USSR " (Geop. Ser), pp. 126-28, (1962).

$\left(^{8}\right)$ Tooley R. D., Spencer T. W., Sagoci II. F., Reflexion and transmission of plane compressional waves. "Geop." pp. 552-70, (1965).

( ${ }^{\ominus}$ Postma G. W., Wave propagation in stratified media. "Geop." P. 780 ff., (1955). 